\title{
DOI:
}

\section{Protein rescue to treat genetic disorders}

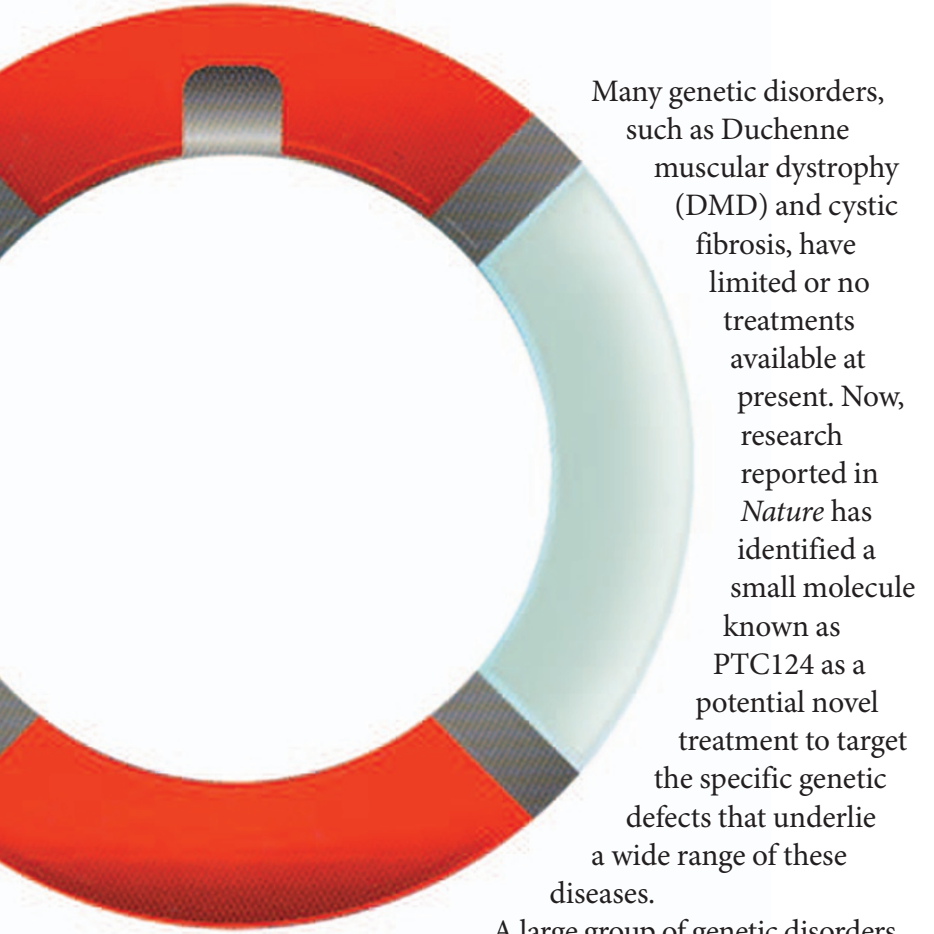

A large group of genetic disorders result from 'nonsense mutations' - DNA alterations that result in premature stop codons within the transcribed mRNA, which lead to

PTC 124 allows

the initial mutation to

be ignored, enabling production of full-length, functional proteins. the early termination of protein translation and the production of a truncated, non-functional protein that is often rapidly degraded. The absence of a specific protein, such as dystrophin in the case of DMD, is the underlying cause of the disease.

Previous studies in cell and animal models have shown that high concentrations of the aminoglycoside gentamicin can promote readthrough of premature stop codons. However, although the production of the respective missing proteins in patients with DMD or cystic fibrosis can be achieved, treatment with gentamicin is associated with poor potency, high toxicity and unfavourable administration methods.

With the aim of identifying potential therapies that might not be limited by such issues, Peltz and colleagues performed high-throughput screening of a small-molecule library comprising 800,000 compounds to find molecules that can suppress premature termination. The chemical scaffolds identified in the screen underwent substantial chemical optimization that ultimately led to the identification of PTC124, which the authors showed can promote readthrough of each of the three known stop codons in stable cell lines that harbour premature nonsense codons. PTC124 does not correct the initial mutation, but merely allows it to be ignored, enabling ribosomal readthrough of premature stop codons in mRNA and the translation and production of full-length, functional proteins.

Next, the authors assessed the potential relevance of the activity of PTC124 to disease treatment. They found that in muscle cells cultured from humans, and in mdx mice (X-linked DMD-mutant mice) that do not produce dystrophin and are a model for DMD, treatment with PTC124 leads to the production of full-length dystrophin. Furthermore, following 2-8 weeks of drug exposure in mdx mice, functional deficits associated with DMD, including decreased striated muscle strength and susceptibility to contractioninduced injury, were partially rescued. These functional effects were associated with increased production of dystrophin, muscle-membrane localization and stabilization of the dystrophin-associated membrane complex.

Importantly, PTC124 was shown to be highly selective for premature stop codons, exerting no effect on normal termination, which is probably due to the existence of mechanistic differences between these two processes. In addition, in contrast to gentamicin, PTC124 exhibited high potency, oral bioavailability, was without nonspecific effects and did not exert obvious toxicity.

The high selectivity of PTC124 for premature stop codons and the notion that nonsense mutations may be the underlying cause of many genetic disorders suggest a broad clinical potential for PTC124. PTC124 is currently undergoing Phase II clinical trials and has so far produced encouraging results for both DMD and cystic fibrosis.

Sarah Crunkhorn

ORIGINAL RESEARCH PAPER Welch, E. et al. PTC124 targets genetic disorders caused by nonsense mutations. Nature $\mathbf{4 4 7}, 87-91$ (2007) 